\title{
The Role of Radio Loud Phase of Nuclear Activity in Galaxy Formation and Evolution
}

\author{
Gabriel A. Ohanian \\ Byurakan Astrophysical Observatory, Armenia \\ email: gohanian@bao.sci.am
}

\begin{abstract}
Key questions, which arise when one tries to clear up a problem of formation and evolution of galaxies, is the question of energy: what is the energetic budget of AGN owing to form galaxies and provide its subsequent development? Hence, for understanding the formation and evolution of galaxies, it is important to estimate the energetic budget of AGN which we try to do involving radio loud phase of nuclear activity.
\end{abstract}

Why radio loud phase of nuclear activity? Radio loud AGN are preferentially hosted by massive early type galaxies and they are usually the brightest galaxies in clusters of galaxies (BCG). BCGs are among the largest and most massive galaxies at all epochs (redshifts) $-\mathrm{M} \sim 10^{10-12} \mathrm{Mo}$ (e.g. Overzier et al. 2009). BCGs are more likely to host a radio AGN than other galaxies of the same stellar or $\mathrm{BH}$ mass. The fraction of radio loud galaxies in BCG increases with mass: the central dominant (cD) galaxies of clusters have a high incidence of radio activity, showing the presence of central FRI radio galaxies in $71 \%$ of the cases (Burns 1990).

In the general scenario of the evolution of powerful radio loud AGNs, GPS sources (Giga-Herts Peaked Spectrum - GPS, linear sizes $<1 \mathrm{kpc}$ ) evolve into CSS sources (Compact Steep Spectrum - CSS, linear sizes between $1 \mathrm{kpc}$ and $20 \mathrm{kps}$ ) and these, into super galactic-size FRI or FRII objects (e.g. O'Dea 1998). The dynamic evolution of the double-lobed radio sources characterized by the total extent of the source, advance speed of the hotspots predicts the increase of the radio power with the linear size of the source in the GPS and CSS phase until they reach the 1-3 kpc size. Then the larger CSS objects should start to slowly decrease their luminosity but the sharp radio power decrease is visible only in the FRI and FRII phase of evolution (e.g. Kaiser \& Best 2007). Finally, after the cut-off of the material supply to the central engine of the galaxy, the sources begin their fading phase. They can come back on the main evolutionary sequence after the re-ignition of the radio activity (Konar et al. 2012). If it is true that GPS and CSS sources are young version of the large radio sources, it means that large scale radio sources host galaxies must live GPS-CSS phase.

High-resolution images of nearby cluster cores taken by Chandra X-ray Observatory show that large amounts of energy are injected into the surrounding medium by powerful outbursts of active galactic nuclei (AGN) lying at the hearts of galaxy clusters (e.g. Nulsen et al. 2005). Non-radiating particles are thought to dominate the energy budget of FRI radio lobes by a factor of $\gg 100$ in some cases, while in FRII radio galaxies, radiating particles are thought to dominate the energy budget (Godfrey \& Shabala 2013). The mean mechanical power of the outburst $\simeq 10^{46} \mathrm{erg} \mathrm{s}^{-1}$, is in the range of quasar luminosities and there is growing evidence that present-day massive elliptical galaxies once hosted the most luminous high-redshift quasars (Hopkins et al. 2007).

If we compare the maximum life time $\left(10^{5} \mathrm{yrs}\right)$ of CSS sources with that of maximum life time of large scale radio sources $\left(10^{8} \mathrm{yrs}\right)$, we will expect $0.1 \%$ fraction in above 
selected catalogs to be CSS sources, assuming that all CSS sources have evolved into large scale radio sources. Since the CSS sources make up $\sim 30 \%$ of the bright extragalactic source population (e.g. O'Dea 1998) then it is natural to suppose the duration of radio sources in the CSS phase will repeat 300 times, before they evolve into large scale radio sources.

There are accumulating evidences that galaxies and AGNs co-exist and it is well known that $\mathrm{cD}$ galaxies are dominated by old stellar populations, up to $1.5 \times 10^{10} \mathrm{yrs}$ old (e.g. Park et al. 2012), and have a high incidence of radio activity. If we suggest that the formation of oldest stars in cD galaxies are closely connected with early activity of nuclear phase (in our case with CSS phase), we can assume, that it would be possible to find some radio sources whose CSS phase of activity will be repeated $3 \times 10^{4}$ times.

The bolometric luminosity of CSS quasars previously detected in X-rays by Chandra is in the range $10^{45}-10^{47} \mathrm{erg} \mathrm{s}^{-1}$ (Migliori 2013). It means that AGN of early type giant galaxies can supply in order of $10^{64}$ erg energy during the whole history of the nucleus. So, energetic budget of AGN of giant EGs must be greater than $10^{64} \mathrm{erg}$.

Assuming the outburst was powered by the gravitational binding energy released by accretion, and adopting mass-energy conversion efficiency is $10 \%$, we find that under these assumptions the black hole grew grater than $5 \times 10^{10}$ Mo. The existence of such ultra massive black holes (UMBHs) in BCGs has recently been confirmed by others (e.g. Hlavacek-Larrondo et al. 2012 and references therein). These results will have important ramifications for the formation and evolution of AGNs.

\section{References}

Burns, J. O. 1990, AJ 99, 14

Godfrey, L. E. H. \& Shabala, S. S. 2013, ApJ 767, 12

Hlavacek-Larrondo, J., Fabian, A. C., Edge, A. C., \& Hogan, M. T. 2012, MNRAS 424, 224

Hopkins, P. F., Hernquist, L., Cox, T. J., Robertson, B., \& Krause, E. 2007, ApJ 669, 67

Kaiser, K. R. \& Best, P. N. 2007, MNRAS 381, 1548

Konar, C., Hardcastle, M. J., Jamrozy, M., Croston, J. H., \& Nandi, S. 2012, MNRAS 424, 1061

Migliori, G., Siemiginowska, A., Kely, B. C., Stawarz, L., Celotti, A., \& Begelman, M. C. 2013, arXiv: 1311.7647

Nulsen, P. E. J., McNamara, B. R., Wise, M. W., \& David, L. P. 2005, ApJ 628, 629

O'Dea, C. P. 1998, PASP 110, 493

Overzier, R. A., Shu, X., Zheng, W., Rettura, A., Zirm, A., et al. 2009, ApJ 704, 548

Park, H. S., Lee, M. G., \& Hwang, H. S. 2012, ApJ 757, 184 